\title{
Revisão do Inventário de Ciúme Romântico: uma análise via modelo de Rasch
}

\author{
Hudson Fernandes Golino ${ }^{1}$ \\ Universidade Estadual de Feira de Santana, Feira de Santana-BA, Brasil \\ Programa de Pós-Graduação em Psicologia da Universidade Salgado de Oliveira, Niterói-RJ, Brasil \\ Lucas de Francisco Carvalho \\ Universidade São Francisco, Itatiba-SP, Brasil \\ Elaine Silva, Eliana Oliveira, Sirneide Santos Musse, Bruna Silva \\ Faculdade de Tecnologia e Ciência, Vitória da Conquista-BA, Brasil
}

\section{RESUMO}

O ciúme romântico é caracterizado como reações exibidas diante da ameaça de perda de um parceiro romântico para um possível rival. Este estudo teve como objetivo revisar os itens do Inventário de Ciúme Romântico (ICR), visando melhorar sua compreensão e testar seu funcionamento em uma amostra da Bahia. Buscou-se verificar evidências de validade da versão modificada do ICR com base na estrutura interna via análise fatorial confirmatória e modelo de Rasch, a partir de dados de 400 participantes de 18 a 76 anos $(M=29,96$; $D P=11,73$ ), sendo $62 \%$ do sexo feminino. Verificou-se o impacto de características individuais na severidade do ciúme romântico. Os dados encontrados corroboram a literatura na área, indicando que o ciúme romântico é composto por diversos fatores, além de um fator geral, o que é refletido também na versão presentemente revisada do ICR. Além disso, outras questões específicas do construto são discutidas no artigo.

Palavras-chave: ciúme; avaliação psicológica; emoções; desconfiança.

\section{ABSTRACT - Revising the Romantic Jealousy Inventory: An analysis via the Rasch model}

Romantic jealousy relates to a series of reactions when facing the threat of loss of a romantic partner to a possible rival. This study aimed to review the items of the Romantic Jealousy Inventory (ICR), in order to improve the their understandability and test its functioning in a sample from Bahia. It sought to verify internal validity evidences via confirmatory factor analysis and the Rasch model. We also looked for the impact of individual features on the severity of romantic jealousy. Therefore, the ICR was applied to 400 participants aged between 18 and 76 years $(M=29.96 ; S D=11.73)$, and $62 \%$ were female. The data corroborate the literature on the matter, indicating that romantic jealousy is composed of several factors, which is also reflected in the currently revised version of the ICR. In addition, other specific construct questions are discussed in the article.

Keywords: jealous; psychological assessment; emotions; distrust.

\section{RESUMEN - Revisión del Inventario de Celos Romántico: un análisis a través del modelo de Rasch}

Celos románticos se refiere a una serie de reacciones cuando se enfrentan a la amenaza de la pérdida de una pareja romántica para un posible rival. Este estudio tuvo como objetivo revisar los artículos del Inventario Romántica los Celos (ICR), con el fin de mejorar la comprensibilidad artículos y probar su funcionamiento en una muestra de Bahía. Se trató de verificar las evidencias de validez interna a través de análisis factorial confirmatorio y el modelo de Rasch. También nos fijamos en el impacto de las características individuales de la gravedad de celos románticos. Por lo tanto, el ICR se aplicó a 400 participantes de edades comprendidas entre 18 y 76 años $(M=29,96 ; S D=11,73)$, y el $62 \%$ eran mujeres. Los datos corroboran la literatura en la zona, lo que indica que los celos románticos se compone de varios factores, que también se refleja en la versión revisada actualmente ICR. Además, otras preguntas constructo específico se discuten en el artículo.

Palabras clave: celos; evaluación psicológica; emociones; desconfianza.

O ciúme é uma reação extremamente comum em seres humanos (Harris, 2005; Kingham, 2004; Mullen \& Martin, 1994), tendo sido citado desde os primeiros registros humanos (e.g., Bíblia) até estudos atuais sob o paradigma científico (e.g., Costa, Sophia, Sanches, Tavares, \&
Zilberman, 2015). Pelas diversas possibilidades de manifestação do ciúme, motivo pelo qual é retratado por vezes como "ciúmes", há entendimentos diferentes sobre esse fenômeno psicológico, sendo considerado como uma emoção social (DeSteno, Bartlett, Braverman, \& Salovey, 2002; 
DeSteno, Valdesolo, \& Bartlett, 2006; Harmon-Jones, Peterson, \& Harris, 2009; Harris, 2003, 2005).

Estudos em psicologia demonstram que o ciúme aparece em diversos contextos (Bueno \& Carvalho, 2012): como tema central de dificuldades em relacionamentos românticos (Marks \& De Silva, 1991; White \& Mullen, 1992); como sintoma de transtornos psiquiátricos (APA, 2000; Dutton, van Ginkel, \& Landolt, 1996; Torres, Ramos-Cerqueira, \& Dias, 1999); ou relacionado a características psicológicas, como Neuroticismo (Carvalho, Bueno, \& Kebleris, 2008), baixa autoestima (DeSteno et al., 2006) e violência (Holtzworth-Munroe, Stuart, \& Hutchinson, 1997).

Em todos os casos, a manifestação mais típica do ciúme se dá nos relacionamentos interpessoais, envolvendo duas pessoas e uma terceira, considerada de maneira mais ou menos consciente como rival, como é o caso das relações amorosas (e.g., De Silva, 1997), entre pais e filhos (e.g., Masciuch \& Kienapple, 1993) ou entre amigos (e.g., Parker, Low, Walker, \& Gamm, 2005). No presente estudo, o foco quanto ao ciúme é o contexto de relacionamentos amorosos, caso em que é nomeado por ciúme romântico (e.g., Buss, Larsen, Westen, \& Semmelroth, 1992).

Estão disponíveis na literatura diversas definições (em ordem alfabética) para o ciúme romântico (e.g., Pines, 1998; De Silva, 1997; DeSteno \& Salovey, 1996; White, 1981); nelas, alguns aspectos relacionados a esse fenômeno são usualmente atribuídos a uma reação complexa e desagradável diante da percepção de uma ameaça por um rival a um relacionamento amoroso considerado importante, envolvendo sentimentos (raiva, ansiedade e tristeza), cognições e comportamentos típicos. Deve-se considerar que, apesar de se caracterizar como um fenômeno universal, dados sugerem que as mulheres apresentam mais ciúme que os homens (Mullen \& Martin, 1994; Sagarin \& Guadagno, 2004). Foge do escopo desta pesquisa uma discussão aprofundada sobre as diferenças entre os sexos na manifestação do ciúme romântico, mas vale ressaltar que os resultados encontrados na área são por vezes contraditórios, mas, no geral, observa-se que as mulheres tendem a apresentar mais reações de ciúme romântico, no que diz respeito à intensidade e à frequência, em comparação ao homem (Carvalho \& Ambiel, no prelo; DeSteno \& Salovey, 1996; Harris, 2005; Sagarin, 2005; Shackelford, Buss, \& Bennett, 2002). Considerando esses aspectos, observa-se que o ciúme romântico é uma reação comum em situações em que a estabilidade do relacionamento amoroso é ameaçada por uma pessoa interpretada como rival (Torres et al., 1999), real ou imaginário, na tentativa de preservar o relacionamento ameaçado (Buss et al., 1992).

Foram encontrados dois instrumentos para a população brasileira (Carvalho et al., 2008; Ramos, Yazawa, \& Salazar, 1994), sendo que um deles apresenta também uma versão revisada (Bueno \& Carvalho, 2012).
Ramos et al. (1994) desenvolveram, em duas versões (uma para homens e uma para mulheres), um instrumento composto por 49 itens que descrevem situações sociais de ciúme; metade dos itens aborda comportamentos ciumentos e metade classifica afirmações sobre comportamentos não ciumentos, devendo ser especificados em uma escala tipo Likert de cinco pontos. Esse instrumento é composto por três fatores ("Não ameaça", "Exclusão" e "Interferência").

$\mathrm{O}$ instrumento desenvolvido por Carvalho et al. (2008), Inventário de Ciúme Romântico (ICR), é composto por 28 itens, que devem ser respondidos em uma escala tipo Likert de 5 pontos, distribuídos em 6 componentes ("Ciúme romântico", "Não ciúme", "Não agressão", "Desconfiança", "Investigação" e "Insegurança"). Os componentes do ICR apresentaram coeficiente de consistência interna variando entre 0,62 e 0,89 $(M=0,76$; $D P=0,09)$. Além disso, foram observadas relações positivas entre "Ciúme Romântico", "Desconfiança", "Investigação" e "Insegurança", isto é, os componentes do Inventário de Ciúme Romântico-Revisado (ICR-R) que dizem respeito à presença do ciúme romântico, com o fator Neuroticismo, que diz respeito à instabilidade de humor, tendência à irritabilidade, à depressividade e à ansiedade (Costa \& MacCrae, 1992).

Apesar das propriedades psicométricas adequadas para os componentes do ICR, algumas inconsistências podem ser apontadas, sugerindo a necessidade de alguma revisão do instrumento (Bueno \& Carvalho, 2012). Entre elas, aponta-se a consistência interna inferior a 0,70 para um dos fatores, além do número excessivo de itens, distribuídos em dois fatores, relacionados com a ausência da manifestação do ciúme romântico. Além disso, deve-se ressaltar que o estudo feito por Carvalho et al. (2008) utilizou exclusivamente uma análise exploratória via componentes principais. A mesma estratégia foi adotada por Bueno e Carvalho (2012) em estudo de revisão do instrumento que identificou um componente de segunda ordem, além dos seis fatores de primeiro nível. Apesar de ser uma estratégia importante nos estudos iniciais, buscando evidências de validade para um instrumento de avaliação, a análise exploratória deve ser acompanhada, em estudos posteriores, de análises confirmatórias. Essa estratégia se deve à necessidade de verificar o ajuste do modelo encontrado aos dados empíricos. Outra limitação diz respeito ao uso de análise de componentes principais. Esse tipo de análise não possui, em sua fundamentação matemática, elementos para estimar o erro de medida. Por esse motivo, não pode ser considerado um modelo de traço latente, o que consequentemente compromete a interpretação dos componentes como variáveis latentes (Borsboom, 2006). Por último, ambos os estudos utilizaram uma amostra específica de um Estado (São Paulo).

As características elencadas sobre os dois estudos prévios com o ICR apontam a necessidade de se utilizar 
modelos confirmatórios, a partir das evidências encontradas nas técnicas exploratórias dos estudos anteriores, assim como de se ampliar a amostra estudada para outras regiões do país. Apontam, ainda, para a importância da aplicação de outros modelos psicométricos, como é o caso da teoria de resposta ao item (TRI), que possibilitem uma análise mais detalhada do instrumento, sobretudo no nível dos itens. Destaca-se aqui que não foram encontrados estudos na literatura científica aplicando modelos da TRI em instrumentos que avaliam ciúme romântico. O objetivo do presente estudo foi revisar os itens do ICR visando melhorar a compreensão sobre os itens e testar seu funcionamento em amostra distinta do estudo inicial com o instrumento. Além disso, partindo da versão modificada do ICR, buscou-se verificar evidências de validade do instrumento com base na estrutura interna por meio da aplicação da análise fatorial confirmatória e do modelo de Rasch para dados dicotômicos. Por último, verificou-se o impacto de características individuais (como idade, sexo, escolaridade, dentre outras) na severidade do ciúme romântico (ou habilidade, ou posição estimada da pessoa traço latente).

\section{Método}

\section{Participantes}

Participaram do presente estudo 400 pessoas de Vitória da Conquista (Bahia), com idades variando entre 18 e 76 anos $(M=29,96 ; D P=11,73)$. Do total de participantes, $62 \%$ são do sexo feminino, $94 \%$ são heterossexuais, $42 \%$ são casados ou moram com algum(a) parceiro(a), $36 \%$ são solteiros e $22 \%$ possuem algum tipo de relacionamento amoroso. Cerca de 39,2\% dos participantes possuem filhos; a mediana do número de relacionamentos que tiveram na vida é de 2 . Com relação à escolaridade dos participantes, $37 \%$ possuem o ensino médio completo, $19 \%$, o ensino superior completo, $12 \%$, o ensino médio incompleto, $10 \%$, o ensino fundamental incompleto, e 7\%, o ensino fundamental completo; $4 \%$ não informaram a escolaridade. No que diz respeito à religião, $56 \%$ são católicos, $30 \%$ são evangélicos e $14 \%$ são de alguma outra religião ou se consideram ateus. A idade mediana da primeira relação sexual é de 16 anos; $4,76 \%$ afirmam ter sofrido algum tipo de abuso sexual; $4,52 \%$ afirmam ter sofrido algum tipo de abuso físico; e $15,91 \%$ afirmam ter sofrido algum tipo de abuso psicológico. A mediana do número de relações sexuais mensais é de $8 ; 31,20 \%$ afirmam terem traído algum(a) parceiro(a) ao longo da vida; e $46,37 \%$ afirmam terem sido traídos por algum(a) parceiro(a). Considerando apenas os participantes que são casados ou que moram com algum(a) parceiro(a) e os que possuem algum outro tipo de relacionamento amoroso, 19,84\% afirmam que já traíram o(a) parceiro(a) atual e 24,5\% afirmam que o(a) parceiro(a) atual já os traiu.

\section{Instrumento}

O primeiro estudo sobre o ICR foi publicado por Carvalho et al. (2008). Por ser uma das primeiras pesquisas em língua portuguesa para avaliar o ciúme romântico, esse estudo original apresentou uma limitação em relação aos itens. Os autores tiveram o cuidado de evitar privilegiar um dos sexos, mas ao fazê-lo acabaram gerando itens cuja leitura era dificultada pela própria estrutura do item. Por exemplo, um dos itens é composto pela seguinte frase: "Sinto raiva quando alguma mulher/algum homem o(a) chama com apelidos carinhosos". No presente trabalho, os itens foram modificados de forma a tornar a leitura mais fluida para o avaliando. Dessa forma, o item exposto foi simplificado para: "Sinto raiva quando alguém chama meu(minha) parceiro(a) com apelidos carinhosos". Essa modificação na estrutura dos itens foi realizada para todos os 27 itens do inventário. Outra modificação implementada diz respeito à conversão de frases negativas em afirmações. Por exemplo, o item "Nunca tentei descobrir a senha do e-mail do meu(minha) parceiro(a)" foi modificada para "Já tentei descobrir a senha do $e$-mail do(da) meu(minha) parceiro(a)". Por último, utilizou-se um formato de resposta dicotômica. No lugar da escala tipo Likert utilizada no estudo original, no presente estudo os participantes tinham de informar se o comportamento descrito no item fazia parte ou não da sua forma de ser (Sim versus Não). É importante apontar que um dos itens originais do instrumento ("Nunca agredi verbalmente outra pessoa que não fosse meu(minha) parceiro(a), por problemas no relacionamento") não foi incluído no presente estudo, devido a um problema no material de coleta de dados (o item saiu consideravelmente ilegível, sendo excluído da presente análise). No entanto, é importante apontar que todos os outros 27 itens ficaram totalmente legíveis no material de coleta de dados.

Além do ICR, foi utilizado um questionário de informações pessoais composto por 18 perguntas abrangendo as seguintes características individuais: idade, sexo, orientação sexual, relacionamento, se tem filhos, número de filhos, quantos relacionamentos teve ao longo da vida, religião, idade da primeira relação sexual, se sofreu abuso sexual, se sofreu abuso físico, se sofreu abuso psicológico, frequência das relações sexuais por mês, escolaridade, se já traiu o parceiro atual, se já traiu algum parceiro, se já foi traído pelo parceiro atual, se já foi traído e com quem mora.

\section{Procedimentos}

Todas as análises realizadas no presente estudo foram implementadas por meio do software de estatística computacional R (R Core Team, 2014). O presente estudo conta com três estratégias de análise de dados. A primeira delas engloba a utilização de análise fatorial confirmatória comparando três principais modelos: unidimensional, hierárquico e bifatorial (Figura 1). O modelo unidimensional 
apresenta apenas 1 variável latente explicando os 27 itens utilizados no estudo. Já o modelo hierárquico apresenta seis variáveis latentes de primeiro nível, explicando os itens do instrumento, e uma variável latente de segundo nível, explicando todas variáveis latentes de primeiro nível. O modelo bifatorial, por sua vez, apresenta seis variáveis latentes de primeiro nível, além de um fator geral também de primeiro nível, todos ortogonais entre si. Os fatores de primeiro nível do modelo hierárquico e do modelo bifatorial foram estipulados a partir da tabela de cargas fatoriais do trabalho de Carvalho et al. (2008).

A Modelo Unidimensional
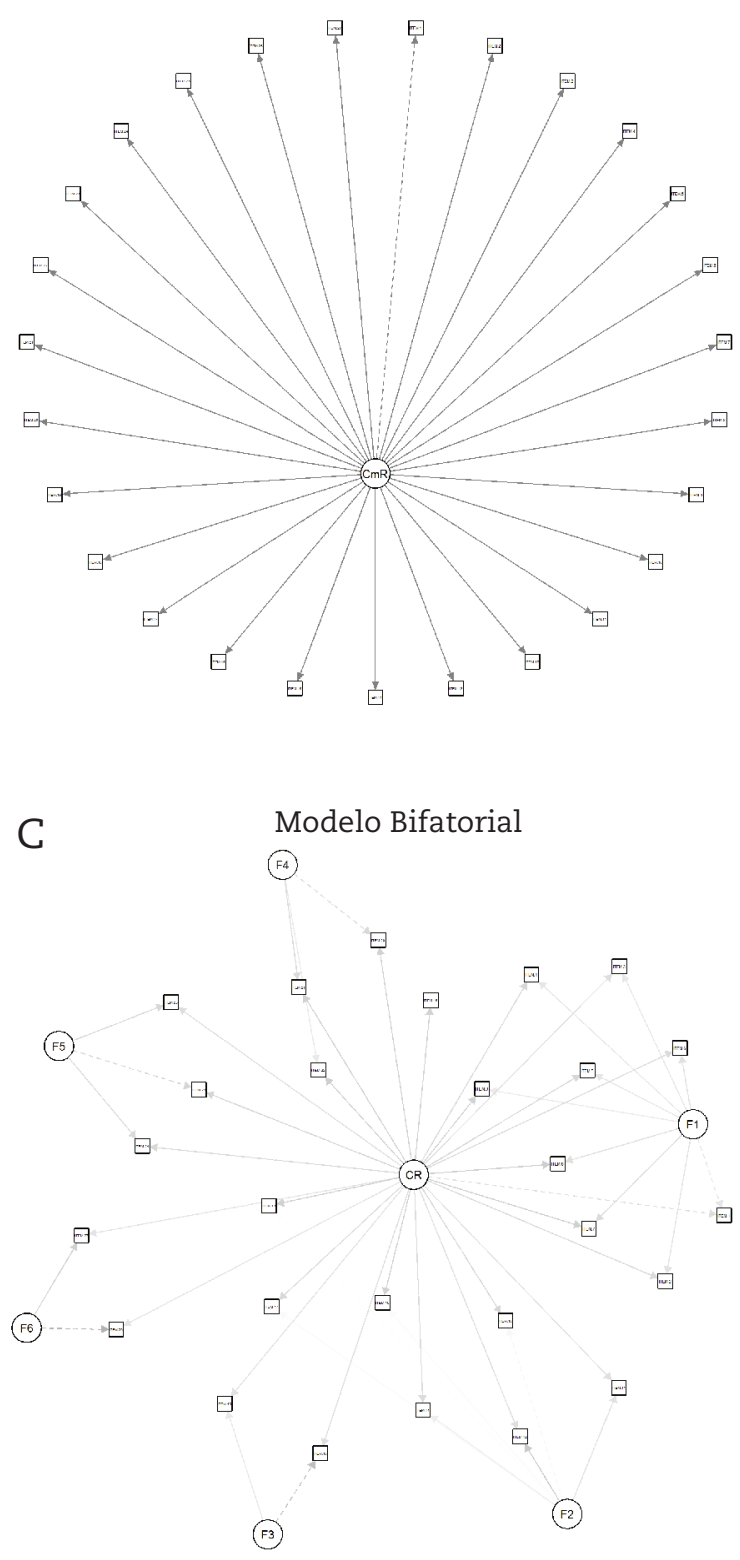

A escolha pela utilização de um modelo bifatorial foi baseada na sugestão de Reise, Moore e Haviland (2010), que argumentam que instrumentos complexos se beneficiam da aplicação de modelos bifatoriais, se comparados com modelos mais tradicionais, como o unidimensional ou os modelos hierárquicos. $\mathrm{O}$ modelo bifatorial permite a estimação de uma variável latente geral, mas também controla a variância que emerge de fatores específicos adicionais. Os autores apontam que o modelo bifatorial é uma estratégia mais apropriada para investigar se os itens de um instrumento avaliam um fator único comum,

B

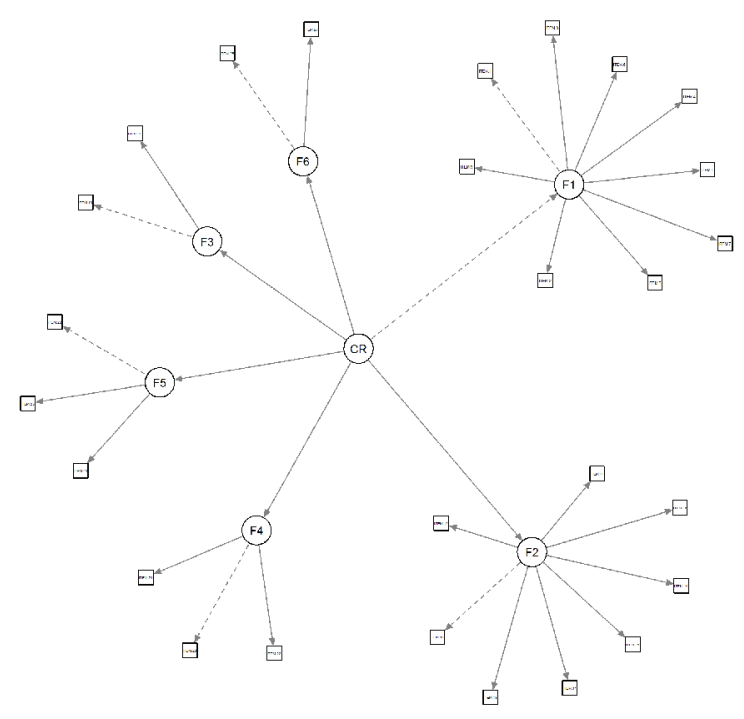

Figura 1. Modelos fatoriais confirmatórios testados: unidimensional (A), hierárquico (B) e bifatorial (C) 
uma vez que o fator geral e os fatores específicos competem pela explicação da variância.

A análise fatorial confirmatória foi implementada por meio do pacote lavaan (Rosseel, 2012), utilizando o estimador WLSM, que é uma versão robusta do weighted least squares. $\mathrm{O}$ ajuste dos dados aos modelos foi verificado por meio do root mean square error of approximation (RMSEA) e do comparative fit index (CFI). Um ajuste adequado aos dados é apontado por meio de um RMSEA igual ou menor que 0,05 (Browne \& Cudeck, 1993) e um CFI igual ou maior que 0,95 (Hu \& Bentler, 1999). As figuras dos modelos fatoriais confirmatórios foram geradas com o auxílio do pacote semPlot (Epskamp, 2014).

A segunda estratégia empregada foi a análise dos itens do ICR por meio do modelo dicotômico de Rasch, utilizando o pacote $e R m$ (Mair, Hatzinger, \& Maier, 2015). De forma a verificar o ajuste dos itens ao modelo dicotômico de Rasch, utilizou-se uma estratégia múltipla (Marais, 2015). Inicialmente, foram verificados os índices outfit mean square e infit mean square (daqui por diante chamados apenas de outfit e infit); depois, foram utilizados o teste da razão de verossimilhança de Andersen (1973) e uma versão ampliada do teste de unidimensionalidade de Martin-Löf (Christensen, Bjørner, Kreiner, \& Petersen, 2002); por último, foram analisadas as curvas características modelada e empírica dos itens, com 24 pontos ao longo do traço latente.

O outfit é um índice de ajuste computado a partir da média do quadrado dos resíduos padronizados de um item. Já o infit é um índice de ajuste que balanceia o resíduo padronizado pela variância desse resíduo, dividindo esse resultado pela média da variância do resíduo (Marais, 2015). Dessa forma, o infit não penaliza os itens que se encontram localizados longe das pessoas no contínuo da variável latente. A interpretação do índice e a predileção pelo seu são baseadas no seguinte pressuposto: se um item se encontra longe da habilidade ${ }^{2}$ das pessoas no contínuo do traço latente, o problema não está na qualidade do item na mensuração do construto, e sim na característica da amostra empregada. Dessa forma, se um item é mais difícil do que a habilidade de todas as pessoas da amostra estudada, o outfit irá penalizar o ajuste do item, mas o infit não. Nesse caso, o outfit aponta a necessidade de encontrar pessoas com maior habilidade para que o item seja aplicado. Em outras palavras, será necessário ampliar a amostra de forma que se tenha pessoas com nível de habilidade no mesmo nível da dificuldade do item. Da mesma forma, se o item é mais fácil do que a habilidade de todas as pessoas da amostra, o outfit irá penalizar o ajuste do item, indicando ser necessário encontrar pessoas com menor habilidade. Tanto o outfit quanto o infit possuem valor esperado de um. Valores inferiores a um indicam que o padrão de resposta das pessoas ao item se ajusta mais do que o esperado pelo modelo. De forma semelhante, valores superiores a um indicam que o padrão de resposta das pessoas ao item se ajusta menos do que o esperado. No entanto, valores de outfit e infit entre 0,7 e 1,3 são considerados adequados (Marais, 2015).

Já o teste da razão de verossimilhança de Andersen testa o princípio subjacente de que, em subgrupos disjuntos arbitrários de pessoas, a estimativa do parâmetro dos itens é a mesma (hipótese nula). Dessa forma, se for possível refutar a hipótese de que a estimativa do parâmetro dos itens é a mesma para $k$ subgrupos, essa será uma forte evidência de desajuste dos itens ao modelo dicotômico de Rasch. A comparação entre as curvas características modelada e empírica dos itens (em múltiplos subgrupos da amostra) é uma estratégia gráfica que possibilita verificar problemas de ajuste, assim como aponta a capacidade discriminativa de cada item (Marais, 2015).

Por último, realizou-se uma análise de regressão linear múltipla para verificar o impacto e o valor significativo das características individuais na estimativa da habilidade das pessoas (escore Rasch). O presente estudo foi conduzido tendo em vista as diretrizes internacionais de ética em pesquisa envolvendo seres humanos (WHO, 2011). É importante apontar que todos os dados utilizados na presente pesquisa estão disponíveis em um repositório on-line para fins de reprodutibilidade dos resultados e para a transparência do processo de investigação científica (http://figshare.com/articles/Dados_da_pesquisa_ sobre_ci_me_rom_ntico/1512812). Encorajamos fortemente os interessados a acessar os dados, disponíveis no formato .csv, e realizar novas análises, ou replicar os resultados encontrados no presente trabalho.

\section{Resultados}

A análise fatorial confirmatória mostrou que o modelo unidimensional $\quad\left[\chi^{2}(324)=952,47 ; \quad p=0,00\right.$; $\mathrm{CFI}=0,92$; RMSEA $=0,07$ (intervalo de confiança de 95\% - IC95\% 0,063-0,077)] não teve um ajuste adequado aos dados, diferentemente do modelo hierárquico testado $\left[\chi^{2}(318)=310,93 ; p=0,00 ; \mathrm{CFI}=0,96 ; \mathrm{RMSEA}=0,05\right.$ (IC95\% 0,038-0,054)]. No entanto, o modelo bifatorial foi o que apresentou o melhor ajuste aos dados $\left[\chi^{2}(297)=426,30 ; p=0,00 ;\right.$ CFI $=0,99 ;$ RMSEA $=0,02$ (IC95\% 0,01-0,03)].

Tais dados corroboram o que foi encontrado por Ramos et al. (1994), Carvalho et al. (2008) e Bueno e Carvalho (2012), casos em que os itens dos instrumentos para avaliação do ciúme romântico se agruparam em mais de um único fator. Além disso, também corroboram a

${ }^{2}$ No presente trabalho, o construto estudado é o ciúme romântico. Por esse motivo, pode ficar estranho ao leitor a utilização do termo habilidade. No entanto, escolheu-se utilizar esse nome, no lugar de severidade, apenas para que fique claro que se trata de uma variável latente estimada. Tanto habilidade quanto severidade são nomes que irão apenas apontar a localização da pessoa no contínuo linear da variável latente investigada. 
literatura, que explicita a composição do ciúme romântico por diversas características correlacionadas, apesar de independentes (e.g., White, 1981). É importante apontar que o modelo bifatorial é uma evidência robusta de que, mesmo com seis variáveis latentes de primeiro nível, o instrumento pode ser considerado satisfatoriamente unidimensional, uma vez que há um fator geral, também de primeiro nível, suficientemente forte. As cargas fatoriais padronizadas do modelo bifatorial podem ser visualizadas na Figura 2.

No que diz respeito aos resultados do modelo dicotômico de Rasch, o outfit variou entre 0,63 e 1,28 $(M=0,95 ; D P=0,14)$, enquanto o infit variou entre 0,84 e $1,20(M=0,99 ; D P=0,08)$. Do ponto de vista dos valores de referências usualmente empregados, o item 24 ("Tenho vontade de contratar um detetive particular para seguir meu(minha) parceiro(a), mas não faria.") apresentou um outfit que indica que o padrão de respostas das pessoas é mais previsível do que o esperado pelo modelo (outfit $=0,63$ ), enquanto o item 1 ("Sinto raiva quando alguém chama meu(minha) parceiro(a) com apelidos carinhosos.") apresentou um outfit que indica que o padrão de respostas das pessoas é menos previsível do que o esperado pelo modelo (outfit $=1,28)$. Apesar de estarem fora do intervalo considerado adequado de ajuste para o outfit, os itens 1 e 24 apresentaram valores adequados de infit (1,20 e 0,87 , respectivamente).

Cargas padronizadas do Modelo Bifatorial

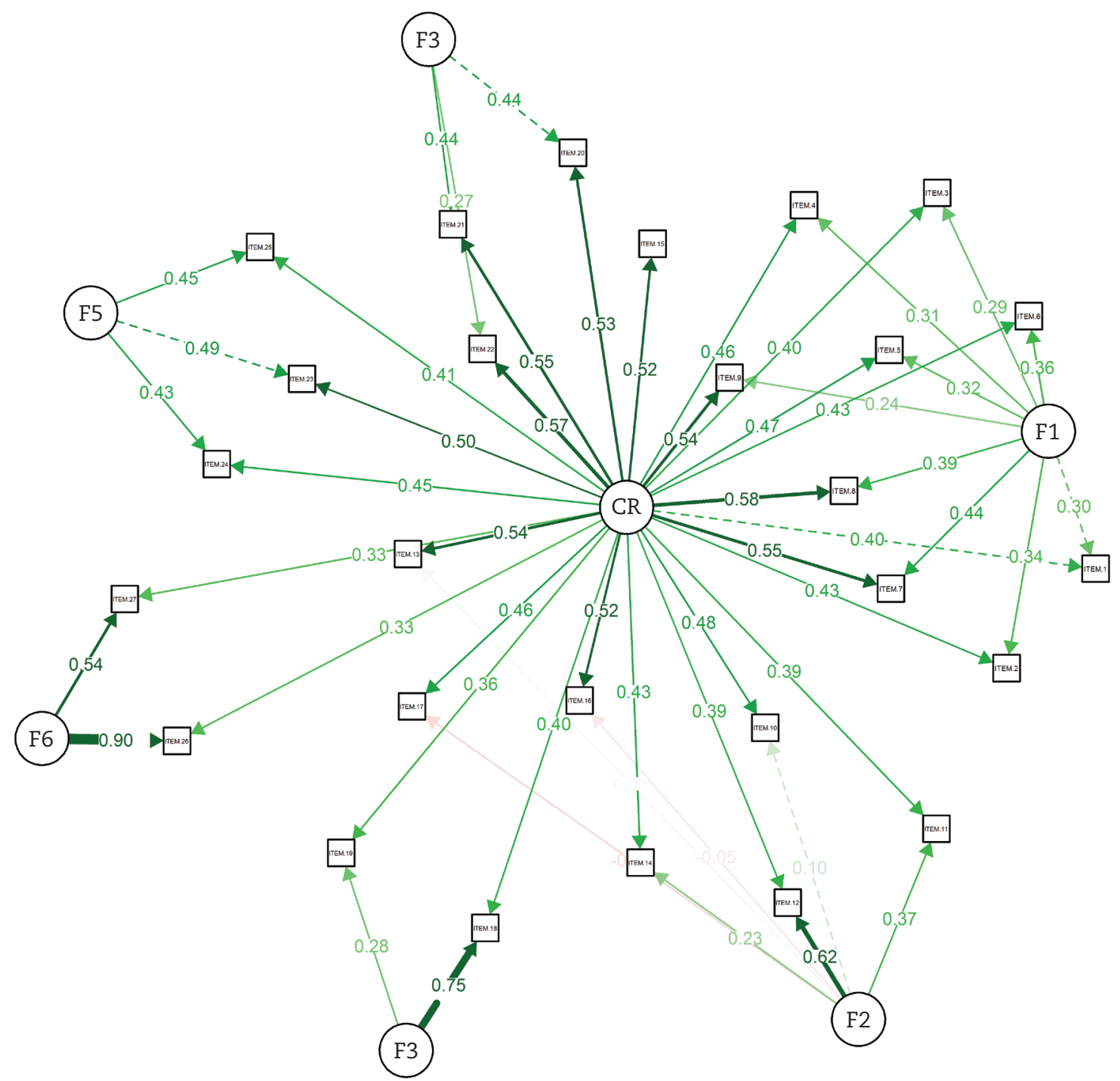

Figura 2. Cargas fatoriais padronizadas do modelo bifatorial do Inventário de Ciúme Romântico 
Como apontado, o outfit é um índice que penaliza o ajuste dos itens que estão mais distantes das habilidades (ou severidade) das pessoas. Justamente os itens 1 e 24 são os itens mais "extremos" em termos de dificuldade, sendo que o primeiro é o mais fácil de ser endossado, e o último, o mais difícil. Esses dados vão de encontro ao relatado por Carvalho et al. (2008) em estudo demonstrando o $5^{\circ}$ fator do ICR, no qual o item 24 se encontra como o menos endossado, provavelmente por apresentar os comportamentos mais atípicos, frequentemente relacionados ao ciúme patológico. Já o item 1 está inserido no primeiro fator do instrumento, que se refere a comportamentos mais típicos de ciúme romântico. Esses dados evidenciam a importância do uso complementar da TRI para compreensão de construtos como o ciúme romântico, já que os componentes desses construtos não necessariamente apresentam ocorrência (presentemente visualizada por meio do nível de endosso) equivalente, tal qual parece ocorrer com o ciúme romântico. Nesse caso, é importante que estudos verifiquem quais são os comportamentos mais usuais, típicos do ciúme romântico, e quais são mais atípicos na população, provavelmente sugerindo a necessidade de intervenções psicológicas. Tanto os índices de ajuste quanto a dificuldade dos itens (neste caso, a dificuldade tem de ser interpretada como dificuldade de endossar o enunciado) estão disponíveis na Tabela 1.

Considerando os dados apresentados na tabela, e contrastando com o que foi encontrado em estudo anterior (Carvalho et al., 2008), observa-se alguma mudança na ordem de endosso (e dificuldade) aos itens. Contudo, os itens dos dois últimos fatores do ICR, Investigação e Insegurança, são consistentemente os menos endossados pelas amostras, de modo que os demais fatores parecem apresentar comportamentos mais tipicamente manifestados de ciúme (Pines, 1998; De Silva, 1997; DeSteno \& Salovey, 1996; White, 1981). Essa informação é de particular relevância na área clínica, indicando ao profissional quais comportamentos podem sinalizar uma maior necessidade interventiva para casos de ciúme romântico.

Ao lado disso, o teste da razão de verossimilhança de Andersen reforçou os achados dos índices outfit e infit, uma vez que apontou que não é possível refutar a hipótese nula de que o parâmetro de dificuldade dos itens é igual para 4 diferentes subgrupos da amostra $(\mathrm{LR}=74,09$; $g l=78 ; p=0,60)$. Do ponto de vista da unidimensionalidade, além da verificação do modelo bifatorial com um fator geral de primeiro nível (ortogonal aos fatores específicos de primeiro nível), outra evidência advém do teste de Martin-Löf, um teste de razão de verossimilhança computado em subgrupos de itens. Para computá-lo, dividimos os itens em 2 subgrupos aleatórios; tal operação apontou que não é possível refutar a hipótese nula da unidimensionalidade dos itens $(\mathrm{LR}=139,38 ; g l=161$; $p=0,89)$. A Figura 3 mostra o chamado mapa de distribuição das variáveis, com a distribuição das habilidades das pessoas (na parte superior) e da dificuldade dos itens (na parte inferior).

Golino e Gomes (2015) apontam como calcular a confiabilidade da medida por meio do modelo dicotômico de Rasch, pela chamada confiabilidade de separação dos itens e das pessoas (Equações 1 e 2):

Confiabilidade de $=\underline{\text { var(Erro Padrão de d)-(MSE d) }}$

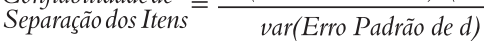

$\begin{aligned} & \text { Confiabilidade de } \\ & \text { Separação das Pessoas }\end{aligned}=\frac{\operatorname{var}(\text { Erro Padrão de b) }-(\text { MSE b })}{\operatorname{var}(\text { Erro Padrão de } b)}$

A forma de se interpretar a confiabilidade de separação é a seguinte: o valor varia entre 0 e 1 , indicando quão bem o padrão de respostas das pessoas, ou o padrão de acerto dos itens, se ajusta à estrutura da medida (Hibbard, Collins, Mahoney, \& Baker, 2010). Dessa forma, a confiabilidade de separação das pessoas nos indica qual a confiança que se tem de que uma pessoa que obtém uma habilidade estimada mais alta de fato possui maior habilidade que outra pessoa que tenha obtido uma habilidade mais baixa. De forma semelhante, a confiabilidade de separação dos itens nos indica qual a confiança que se tem de que um item de dificuldade estimada mais alta de fato possui maior dificuldade que outro item de dificuldade estimada mais baixa (Golino \& Gomes, 2015). A confiabilidade de separação dos itens do ICR foi de 0,96 , e a confiabilidade de separação das pessoas, de 0,89 . Ressalta-se que esses dados sugerem a capacidade discriminativa da versão presentemente utilizada do ICR.

As Figuras 4 e 5 apresentam as curvas características dos itens (modeladas, com linha contínua preta, e empíricas, com círculos cinzas) do ICR. A partir dessas figuras é possível identificar graficamente o ajuste dos itens, assim como aqueles que possuem maior discriminação. Os 24 pontos mostram diferentes desempenhos médios (em termos de endosso aos itens) ao longo do traço latente. Apenas em alguns itens (5, 8, 9, 10 e 11) é possível observar uma reversão do endosso médio com a intensificação do traço latente. No entanto, são reversões pequenas em um intervalo bastante curto do traço latente, não representando um problema para o ajuste do item, o que é refletido pelos seus índices de ajuste. Os itens com maior discriminação $(11,12,14,17,19,23$, 24,25 e 27) estão entre aqueles que possuem as maiores dificuldades de endosso.

Tanto os índices de separação quanto as Figuras 4 e 5 indicam que o conjunto de itens que compõe o ICR é capaz de diferenciar pessoas nos diferentes níveis no construto latente. Isto é, de fato, pessoas em diferentes níveis no instrumento são distintas em termos de construto. 
Tabela 1

Descritor dos itens, outfit, infit e dificuldade

\begin{tabular}{|c|c|c|c|c|}
\hline Item & Descritor & Outfit MSQ & Infit MSQ & Dificuldade \\
\hline 1 & $\begin{array}{l}\text { Sinto raiva quando alguém chama meu(minha) parceiro(a) com } \\
\text { apelidos carinhosos. }\end{array}$ & 1,28 & 1,20 & $-1,44$ \\
\hline 2 & $\begin{array}{l}\text { Quando vejo meu(minha) parceiro(a) conversando com alguém } \\
\text { com quem já se relacionou, me sinto muito insegura(o). }\end{array}$ & 1,16 & 1,09 & $-1,24$ \\
\hline 3 & $\begin{array}{l}\text { Quando meu(minha) parceiro(a) } \\
\text { olha para alguém, me sinto insegura(o). }\end{array}$ & 1,04 & 1,00 & $-0,27$ \\
\hline 4 & $\begin{array}{l}\text { Quando alguém liga para meu parceiro(a) e fica conversando } \\
\text { horas, fico desconfiada(o). }\end{array}$ & 1,06 & 1,05 & $-1,16$ \\
\hline 5 & $\begin{array}{l}\text { Fico desconfiada(o) quando } \\
\text { meu(minha) parceiro(a) elogia alguém }\end{array}$ & 1,10 & 1,09 & $-0,47$ \\
\hline 6 & Quando meu(minha) parceiro(a) abraça alguém sinto raiva. & 0,97 & 1,01 & $-0,01$ \\
\hline 7 & $\begin{array}{l}\text { Me sinto insegura(o) quando } \\
\text { meu(minha) parceiro(a) viaja sozinho(a). }\end{array}$ & 0,78 & 0,84 & $-0,73$ \\
\hline 8 & $\begin{array}{l}\text { Sinto desconfiança quando } \\
\text { meu(minha) parceiro(a) viaja sozinho(a). }\end{array}$ & 0,78 & 0,84 & $-0,63$ \\
\hline 9 & $\begin{array}{l}\text { Quando posso, arrumo uma forma de ver as ligações recebidas/ } \\
\text { feitas e/ou as mensagens no celular do meu(minha) parceiro(a). }\end{array}$ & 0,99 & 0,95 & $-0,93$ \\
\hline 10 & Já tentei descobrir a senha do e-mail do meu(minha) parceiro(a). & 0,93 & 0,96 & 0,27 \\
\hline 11 & Ouço as conversas do meu parceiro(a) pela linha telefônica. & 1,18 & 1,01 & 0,93 \\
\hline 12 & $\begin{array}{l}\text { Julgo que há problema quando meu(minha) parceiro(a) olha } \\
\text { para alguém atraente na TV ou cinema. }\end{array}$ & 0,83 & 0,98 & 1,06 \\
\hline 13 & $\begin{array}{l}\text { Já tive vontade de procurar por "coisas suspeitas" no quarto do } \\
\text { meu(minha) parceiro(a) ou em outro local referente a ele(a). }\end{array}$ & 1,00 & 1,02 & $-0,19$ \\
\hline 14 & $\begin{array}{l}\text { Julgo que há problema quando vou com meu(minha) parceiro(a) } \\
\text { a lugares públicos como clubes, praias, etc. onde existam } \\
\text { pessoas trajando roupas de banho. }\end{array}$ & 0,92 & 1,04 & 0,41 \\
\hline 15 & $\begin{array}{l}\text { Julgo que há problemas quando meu(minha) parceiro(a) } \\
\text { cumprimenta certas pessoas. }\end{array}$ & 1,06 & 0,98 & $-0,01$ \\
\hline 16 & $\begin{array}{l}\text { Quando desconfio do(a) meu(minha) } \\
\text { parceiro(a) tenho vontade de segui-lo(a) }\end{array}$ & 1,01 & 0,99 & 0,05 \\
\hline 17 & Já pedi para vigiarem o(a) meu(minha) parceiro(a) para mim. & 0,99 & 1,01 & 0,48 \\
\hline 18 & $\begin{array}{l}\text { Já tive vontade de agredir fisicamente outra pessoa, que } \\
\text { não fosse meu(minha) parceiro(a), por problemas no } \\
\text { relacionamento. }\end{array}$ & 0,90 & 0,99 & 0,31 \\
\hline 19 & $\begin{array}{l}\text { Já agredi fisicamente alguém que não meu(minha) parceiro(a), } \\
\text { por problemas no relacionamento. }\end{array}$ & 1,00 & 1,03 & 0,64 \\
\hline 20 & $\begin{array}{l}\text { Quando meu(minha) parceiro(a) não atende o celular, penso } \\
\text { que ele(a) pode estar fazendo algo que me desagrade. }\end{array}$ & 0,93 & 1,02 & $-0,49$ \\
\hline 21 & $\begin{array}{l}\text { Quando meu(minha) parceiro(a) diz que vai me ligar e não liga, } \\
\text { sinto desconfiança. }\end{array}$ & 0,91 & 0,99 & $-0,49$ \\
\hline 22 & $\begin{array}{l}\text { Desconfio quando meu(minha) } \\
\text { parceiro(a) sai sem falar para onde vai. }\end{array}$ & 0,87 & 0,97 & $-0,54$ \\
\hline 23 & $\begin{array}{l}\text { Eu contrataria um detetive particular } \\
\text { para seguir meu(minha) parceiro(a). }\end{array}$ & 0,80 & 0,89 & 0,72 \\
\hline 24 & $\begin{array}{l}\text { Tenho vontade de contratar um detetive particular para seguir } \\
\text { meu(minha) parceiro(a), mas não faria. }\end{array}$ & 0,63 & 0,85 & 1,23 \\
\hline 25 & Quando desconfio do meu(minha) parceiro(a) o(a) sigo. & 0,74 & 0,88 & 1,09 \\
\hline 26 & $\begin{array}{l}\text { Fico perguntando para as pessoas que conhecem meu(minha) } \\
\text { parceiro(a) se ele(a) gosta realmente de mim. }\end{array}$ & 0,81 & 1,04 & 0,81 \\
\hline 27 & $\begin{array}{l}\text { Tenho vontade de perguntar para os amigos do meu(minha) } \\
\text { parceiro(a) se ele(a) gosta realmente de mim. }\end{array}$ & 1,03 & 1,07 & 0,61 \\
\hline
\end{tabular}

Nota: $\mathrm{MSQ}=$ mean square. 


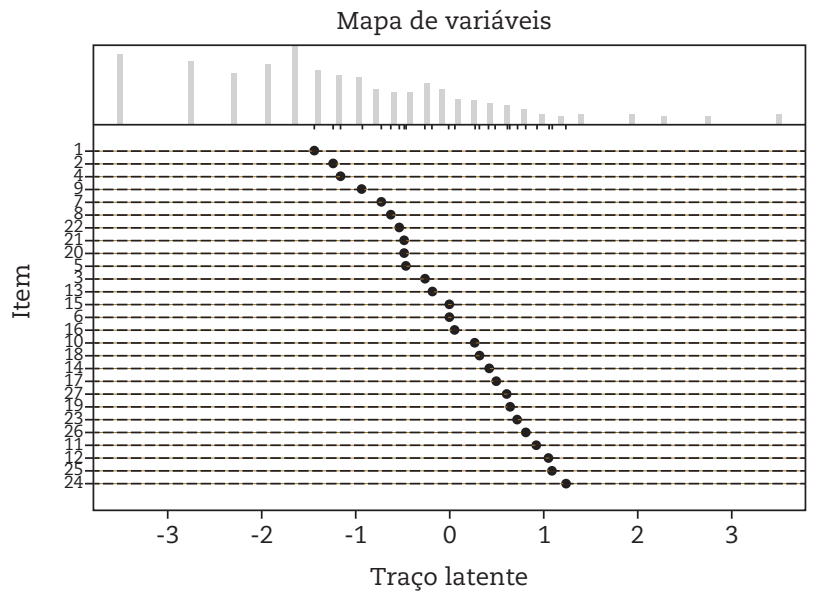

Figura 3. Mapa de distribuição das habilidades e dificuldades
Os dados encontrados em estudos anteriores (Bueno \& Carvalho, 2012; Carvalho et al., 2008) já apontavam essa tendência, mas os dados mais diretamente relacionados a essa capacidade discriminativa são inéditos (presente estudo). Ainda nesse sentido, observa-se que o ICR se torna uma ferramenta mais discriminativa, sobretudo a partir da aplicação de itens menos endossados (isto é, mais difíceis). Esses dados são coerentes com a literatura na área do ciúme romântico, que sugere que esse seja um fenômeno universal e frequente (Harris, 2005; Kingham, 2004; Mullen \& Martin, 1994), mas que também pode apresentar reações menos típicas, como, por exemplo, comportamentos investigativos (Carvalho et al., 2008).

A última parte da análise de dados envolveu o emprego de uma análise de regressão linear (escore Rasch) para verificar as características individuais que mais

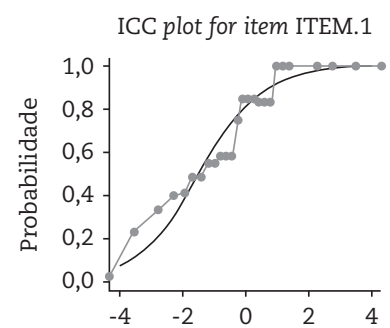

Contínuo da variável latente

ICC plot for item ITEM.5

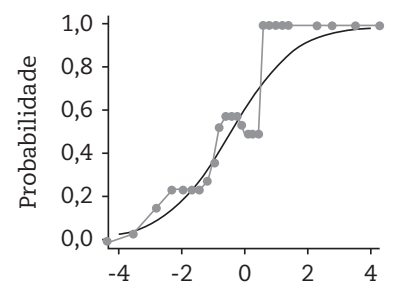

Contínuo da variável latente

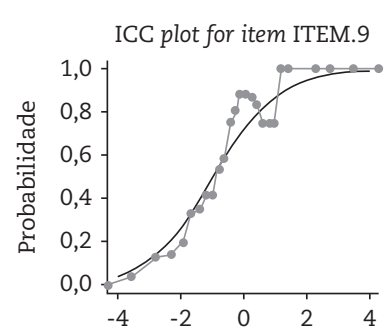

Contínuo da variável latente

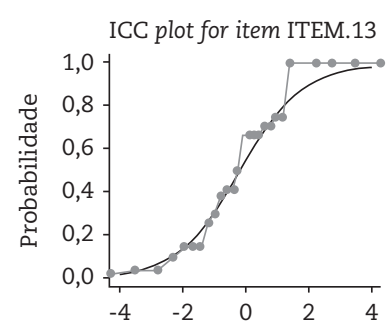

Contínuo da variável latente

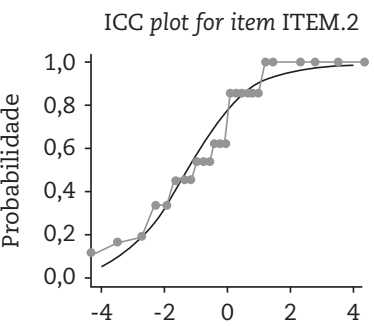

Contínuo da variável latente

ICC plot for item ITEM. 6

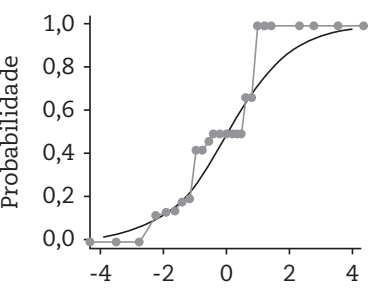

Contínuo da variável latente

ICC plot for item ITEM.10

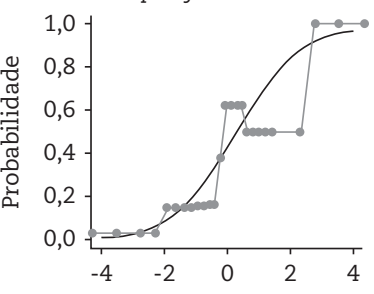

Contínuo da variável latente

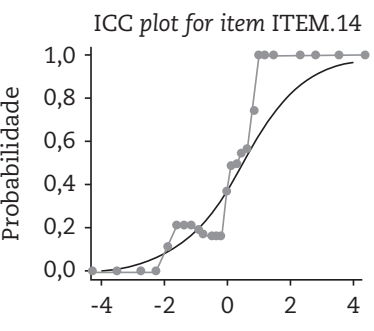

Contínuo da variável latente
ICC plot for item ITEM.3

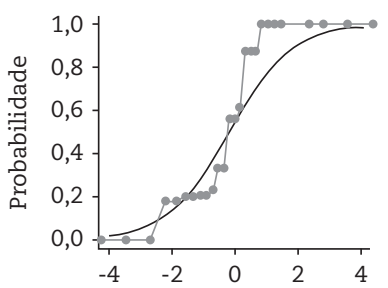

Contínuo da variável latente

ICC plot for item ITEM.7

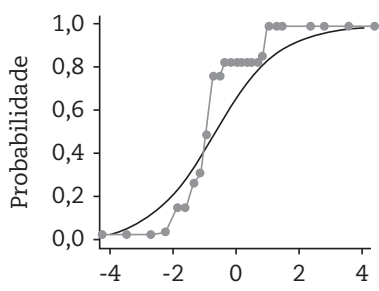

Contínuo da variável latente

ICC plot for item ITEM.11

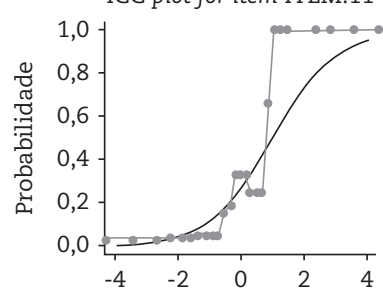

Contínuo da variável latente

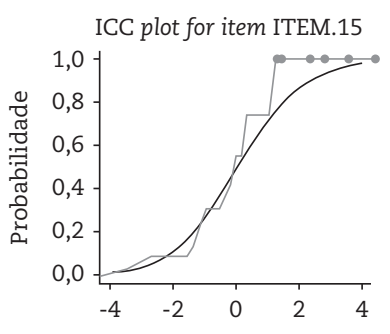

Contínuo da variável latente

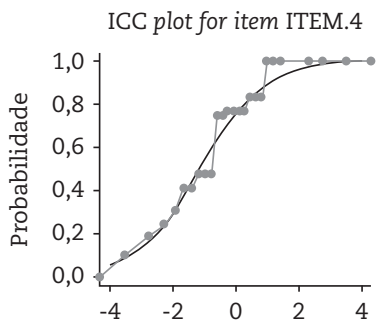

Contínuo da variável latente

ICC plot for item ITEM.8

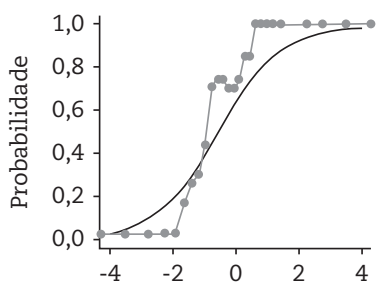

Contínuo da variável latente

ICC plot for item ITEM.12

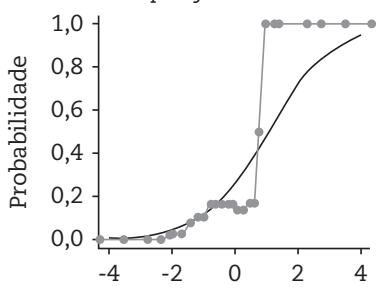

Contínuo da variável latente

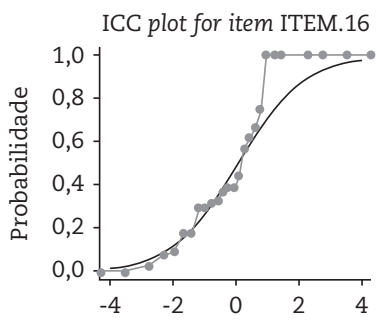

Contínuo da variável latente

Figura 4. Curvas características dos itens de 1 a 16 

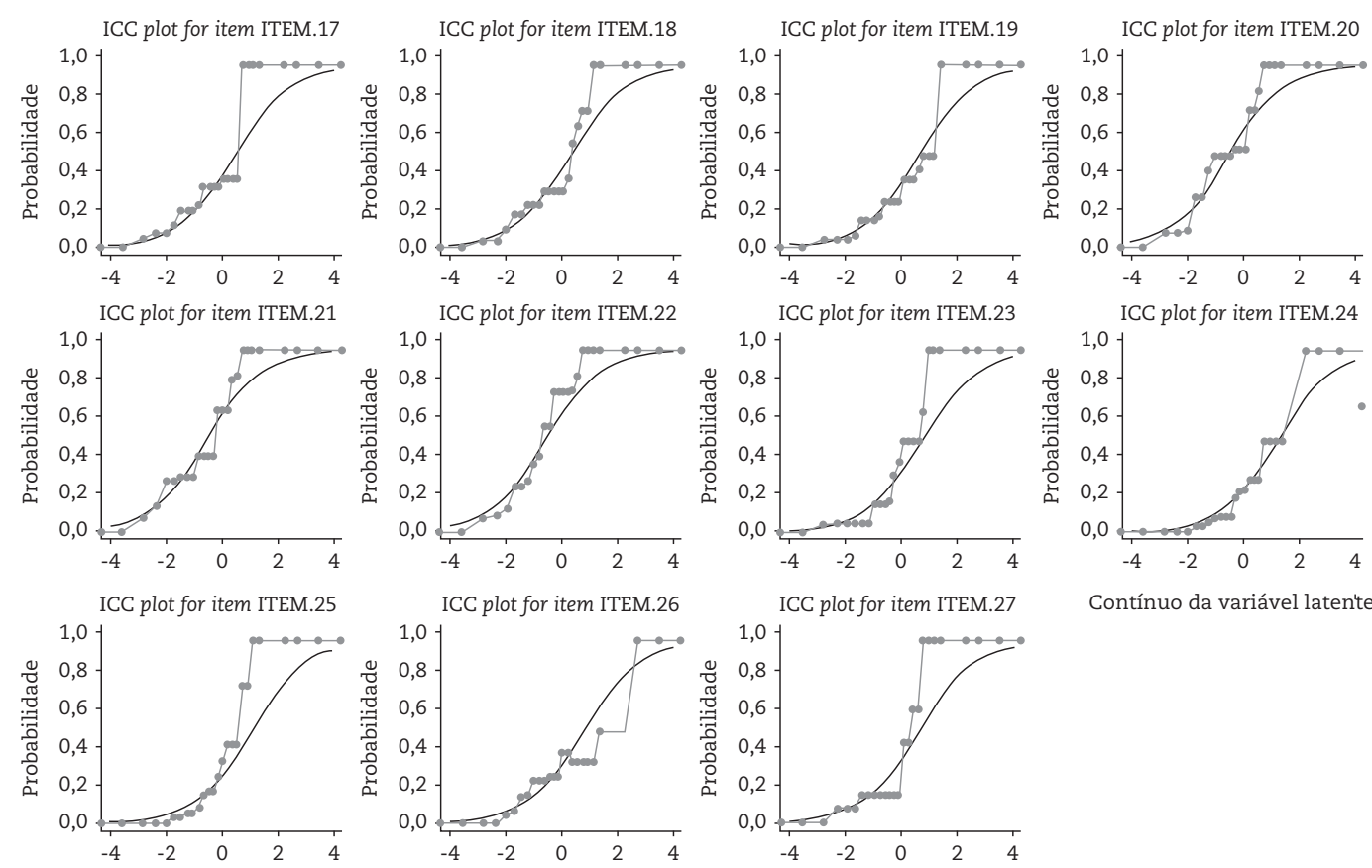

Contínuo da variável laten'te

Contínuo da variável latente

Contínuo da variável latente

Contínuo da variável latente

Figura 5. Curvas características dos itens de 17 a 27
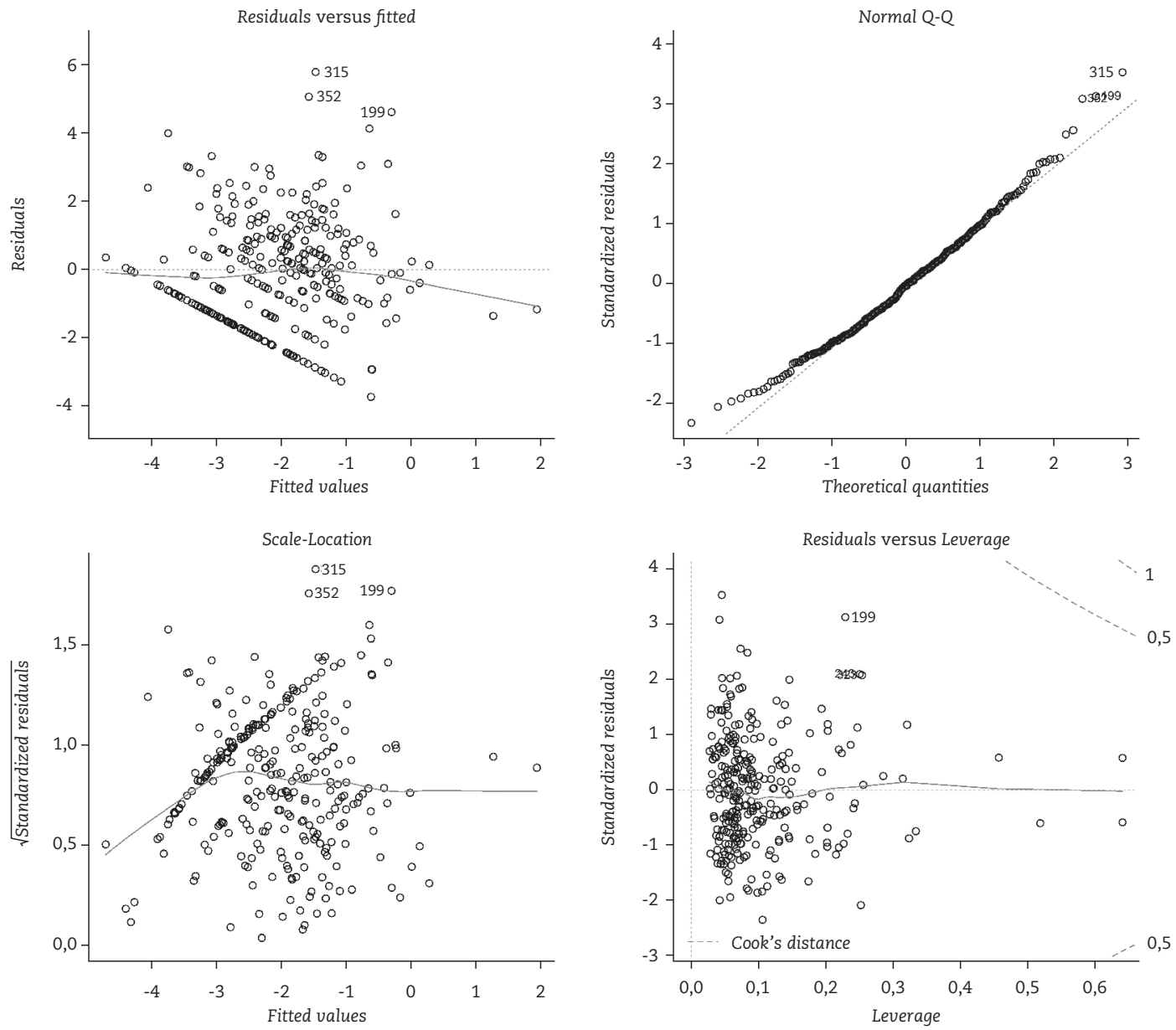

Figura 6. Gráfico diagnóstico da regressão linear 
impactam a estimativa da habilidade (ou severidade) das pessoas. A Figura 6 mostra os gráficos diagnósticos para a regressão linear apontando que há elementos suficientes para sustentar a utilização da técnica.

São preditores significativos do escore Rasch do ciúme romântico o sexo feminino, ter um relacionamento sério ou ser solteiro(a), a idade da primeira relação sexual e o abuso físico (Tabela 2). Dentre essas variáveis, apenas a idade da primeira relação sexual possui um coeficiente negativo, indicando que cada ano diminui em -0,073 o escore Rasch de ciúme romântico.

Ressalta-se que os dados apresentados na Tabela 2 visam complementar tanto o conhecimento acerca do funcionamento do ICR quanto incrementar a literatura na área do ciúme romântico. Especificamente em relação à variável sexo, é coerente com a literatura (DeSteno \& Salovey, 1996; Harris, 2005; Sagarin, 2005; Shackelford, Buss, \& Bennett, 2002) apontar que ser do sexo feminino apresenta capacidade preditiva significativa, já que, no geral, observa-se que as mulheres tendem a apresentar de maneira mais intensa e frequente as reações típicas de ciúme romântico. Contudo, no que concerne às demais variáveis, pesquisas investigando dados similares são escassas na literatura, dificultando o atual aprofundamento dos achados. Nesse sentido, pesquisas futuras mais direcionadas a compreender modelos de

Tabela 2

Coeficientes da regressão linear

\begin{tabular}{|c|c|c|c|c|}
\hline & Estimativa & $\mathrm{DP}$ & $t$ & $\operatorname{Pr}(>|t|)$ \\
\hline (Intercepto) & $-2,95$ & 1,81 & $-1,63$ & 0,10432 \\
\hline Sexo=feminino & 0,95 & 0,23 & 4,07 & $0,00000^{* * *}$ \\
\hline Heterossexual & $-0,20$ & 1,77 & $-0,11$ & 0,91169 \\
\hline Homossexual & 0,26 & 1,88 & 0,14 & 0,89048 \\
\hline Bissexual & $-0,56$ & 2,27 & $-0,25$ & 0,80668 \\
\hline Relacionamento sério & 0,81 & 0,33 & 2,45 & $0,01481^{* *}$ \\
\hline Solteiro(a) & 0,67 & 0,31 & 2,16 & $0,03189^{* *}$ \\
\hline Viúvo(a) & 0,18 & 0,96 & 0,19 & 0,85334 \\
\hline Tem filhos & 0,03 & 0,39 & 0,07 & 0,94722 \\
\hline Quantos filhos & 0,01 & 0,17 & 0,04 & 0,96831 \\
\hline Quantos relacionamentos teve & 0,15 & 0,08 & 1,81 & 0,07108 \\
\hline Religião=evangélica & 0,33 & 0,24 & 1,35 & 0,17798 \\
\hline Religião=espírita & $-0,54$ & 0,51 & $-1,06$ & 0,29205 \\
\hline Ateu/agnóstico & $-1,00$ & 0,55 & $-1,80$ & 0,07283 \\
\hline Religião=outra & 0,93 & 0,49 & 1,91 & 0,05733 \\
\hline Idade da primeira relação sexual & $-0,07$ & 0,02 & $-3,27$ & $0,00124^{* *}$ \\
\hline Sofreu abuso sexual & $-0,49$ & 0,66 & $-0,74$ & 0,45986 \\
\hline Sofreu abuso físico & 2,04 & 0,76 & 2,67 & $0,00798^{*}$ \\
\hline Sofreu abuso psicológico & 0,35 & 0,38 & 0,93 & 0,35427 \\
\hline Frequência das relações sexuais por mês & 0,02 & 0,01 & 1,10 & 0,27209 \\
\hline Escolaridade1 & 0,65 & 0,59 & 1,09 & 0,27724 \\
\hline Escolaridade2 & $-0,48$ & 0,64 & $-0,75$ & 0,45273 \\
\hline Escolaridade3 & $-0,32$ & 0,61 & $-0,53$ & 0,59747 \\
\hline Escolaridade4 & 0,89 & 0,55 & 1,61 & 0,10892 \\
\hline Escolaridade5 & 0,86 & 0,61 & 1,39 & 0,16481 \\
\hline Escolaridade6 & 0,59 & 0,54 & 1,10 & 0,27324 \\
\hline Já traiu parceiro atual & $-0,08$ & 0,28 & $-0,28$ & 0,77891 \\
\hline Já traiu algum parceiro & 0,35 & 0,28 & 1,28 & 0,20235 \\
\hline Já foi traído pelo parceiro atual & 0,28 & 0,19 & 1,45 & 0,1479 \\
\hline Já foi traído & 0,00 & 0,16 & $-0,01$ & 0,99058 \\
\hline
\end{tabular}

Nota: DP=desvio padrão; ${ }^{*} p=0,05 ;{ }^{* *} p=0,001 ;{ }^{* * *} p=0,0001$. 
ciúme romântico devem buscar replicar esses achados, na tentativa de entender relações mais explícitas com o ciúme romântico.

\section{Considerações Finais}

O presente estudo buscou desenvolver uma nova versão do ICR, com o objetivo de melhorar a compreensão sobre seus itens, além de verificar seu funcionamento em amostras distintas dos estudos anteriormente realizados. Os dados encontrados sugerem que a versão presentemente estabelecida tenha um funcionamento adequado do ponto de vista da psicometria, incluindo índices de fidedignidade e evidências de validade com base na estrutura interna.

Os dados aqui apresentados devem ser ponderados considerando as restrições da amostra, do modo de coleta de dados e dos modelos matemáticos utilizados. Futuros estudos devem buscar replicar os achados da presente pesquisa, investigando a persistência ou não dos resultados em amostras distintas. Além disso, é necessário verificar de maneira minuciosa as relações entre o ciúme romântico e as variáveis sociodemográficas inicialmente exploradas neste estudo.

\section{Referências}

Andersen, E. B. (1973). A goodness of fit test for the Rasch model. Psychometrika, 38, 123-140.

American Psychiatric Association. (2000). Diagnostic and Statistical Manual of Mental Disorders: DSM-IV-TR. Arlington: APA.

Borsboom, D. (2006). The attack of the psychometricians. Psychometrika, 71(3), 425-440.

Browne, M. W., \& Cudeck, R. (1993). Alternative ways of assessing model fit. Em K. A. Bollen \& J. Long, Testing structural equation models. (pp. 136-162). Newbury Park: Sage.

Bueno, J. M. H., \& Carvalho, L. de F. (2012). Um estudo de revisão do Inventário de Ciúme Romântico (ICR). Psicologia: Reflexão E Crítica, 25(3), 435-444. doi: 10.1590/S0102-79722012000300003

Buss, D. M., Larsen, R. J., Westen, D., \& Semmelroth, J. (1992). Sex differences in jealousy: Evolution, Physiology, and Psychology. Psychological Science, 3(4), 251-255. doi: 10.1111/j.1467-9280.1992.tb00038.x

Carvalho, L. de F., \& Ambiel, R. A. M. (no prelo). Diferenças entre os sexos no ciúme romântico: um estudo brasileiro. Avances en Psicología Latinoamericana.

Carvalho, L. de F., Bueno, J. M. H., \& Kebleris, F. (2008). Estudos psicométricos preliminares do Inventário de Ciúme Romântico: ICR. Avaliação Psicológica, 7(3), 335-346.

Christensen, K. B., Bjørner, J. B., Kreiner, S., \& Petersen, J. H. (2002). Testing unidimensionality in polytomous Rasch models. Psychometrika, 67(4), 563-574.

Costa, A. L., Sophia, E. C., Sanches, C., Tavares, H., \& Zilberman, M. L. (2015). Pathological jealousy: Romantic relationship characteristics, emotional and personality aspects, and social adjustment. Journal of Affective Disorders, 174, 38-44. doi: 10.1016/j.jad.2014.11.017

Costa, P., \& MacCrae, R. (1992). Revised NEO personality inventory (NEO PI-R) and NEO five-factor inventory (NEO FFI): Professional manual. Recuperado de https://scholar.google.com/scholar?hl=pt-BR\&as_sdt=0,5\&cluster $=8500625450659321461 \# 0$

De Silva, P. (1997). Jealousy in couple relationships: Nature, assessment and therapy. Behaviour Research and Therapy, 35(11), 973-985.

DeSteno, D. A., \& Salovey, P. (1996). Evolutionary Origins of sex differences in jealousy? Questioning the "Fitness" of the Model. Psychological Science, 7(6), 367-372. doi: 10.1111/j.1467-9280.1996.tb00391.x

DeSteno, D., Bartlett, M. Y., Braverman, J., \& Salovey, P. (2002). Sex differences in jealousy: Evolutionary mechanism or artifact of measurement? Journal of Personality and Social Psychology, 83(5), 1103-1116. Recuperado de http://www.ncbi.nlm.nih.gov/ pubmed/12416915

DeSteno, D., Valdesolo, P., \& Bartlett, M. Y. (2006). Jealousy and the threatened self: Getting to the heart of the green-eyed monster. Journal of Personality and Social Psychology, 91(4), 626-41. doi: 10.1037/0022-3514.91.4.626

Dutton, D. G., van Ginkel, C., \& Landolt, M. A. (1996). Jealousy, intimate abusiveness, and intrusiveness. Journal of Family Violence, 11(4), 411-423. doi: 10.1007/BF02333425

Epskamp, S. (2014). semPlot: Path diagrams and visual analysis of various SEM packages' output. Recuperado de http://CRAN.R-project.org/ package $=$ semPlot

Golino, H. F., \& Gomes, C. M. (2015). Investigando estágios de desenvolvimento do raciocínio indutivo usando a análise fatorial confirmatória, o Modelo Logístico Simples de Rasch e o modelo de teste logístico linear (Rasch Estendido). Em H. F. Golino, C. M. Gomes, A. Amantes \& G. Coelho, Psicometria Contemporânea: compreendendo os Modelos Rasch. (pp. 283-331). São Paulo: Casa do Psicólogo/Pearson.

Harmon-Jones, E., Peterson, C. K., \& Harris, C. R. (2009). Jealousy: Novel methods and neural correlates. Emotion, 9(1), 113-117. doi: $10.1037 / \mathrm{a} 0014117$

Harris, C. R. (2003). A review of sex differences in sexual jealousy, including self-report data, psychophysiological responses, interpersonal violence, and morbid jealousy. Personality and Social Psychology Review, 7(2), 102-128.

Harris, C. R. (2005). Male and female jealousy, still more similar than different: Reply to Sagarin (2005). Personality and Social Psychology Review, 9(1), 76-86. doi: 10.1207/s15327957pspr0901_6

Hibbard, J., Collins, P., Mahoney, E., \& Baker, L. (2010). The development and testing of a measure assessing clinician beliefs about patient self-management. Health Expectations: An International Journal of Public Participation in Health Care, 13(1), 65-72. 
Holtzworth-Munroe, A., Stuart, G. L., \& Hutchinson, G. (1997). Violent versus nonviolent husbands: Differences in attachment patterns, dependency, and jealousy. Journal of Family Psychology, 11(3), 314-331.

Hu, L. T., \& Bentler, P. (1999). Cutoff criteria for fit indexes in covariance structure. Structural Equation, 6(1), 1-55.

Kingham, M. (2004). Aspects of morbid jealousy. Advances in Psychiatric Treatment, 10(3), 207-215. doi: 10.1192/apt.10.3.207

Mair, P., Hatzinger, R., \& Maier, M. J. (2015). Modelos Rasch estendidos: apresentando o pacote eRm. Em H. F. Golino, C. M. Gomes, A. Amantes \& G. Coelho, Psicometria Contemporânea: Compreendendo os Modelos Rasch. (pp. 209-237). São Paulo: Casa do Psicólogo/Pearson.

Marais, I. (2015). Ajuste das respostas ao modelo Rasch. Em H. F. Golino, C. M. Gomes, A. Amantes \& G. Coelho, Psicometria Contemporânea: Compreendendo os Modelos Rasch. (pp. 185-207). São Paulo: Casa do Psicólogo/Pearson.

Marks, M., \& De Silva, P. (1991). Multi-faceted treatment of a case of morbid jealousy. Sexual and Marital Therapy, 6(1), 71-78. doi: $10.1080 / 02674659108408356$

Masciuch, S., \& Kienapple, K. (1993). The emergence of jealousy in children 4 months to 7 years of age. Journal of Social and Personal Relationships, 10(3), 421-435. doi: 10.1177/0265407593103008

Mullen, P. E., \& Martin, J. (1994). Jealousy: A community study. The British Journal of Psychiatry, 164(1), 35-43. doi: 10.1192/bjp.164.1.35

Parker, J. G., Low, C. M., Walker, A. R., \& Gamm, B. K. (2005). Friendship jealousy in young adolescents: Individual differences and links to sex, self-esteem, aggression, and social adjustment. Developmental Psychology, 41(1), 235-250. doi: 10.1037/0012-1649.41.1.235

Pines, A. M. (1998). Romantic jealousy: Causes, symptoms, cures. New York: Routledge.

Ramos, A. L. M., Yazawa, S. A. K., \& Salazar, A. F. (1994). Desenvolvimento de uma escala de ciúme romântico. Psicologia: Teoria e Pesquisa, 10(3), 439-451.

R Core Team. (2014). R: A language and environment for statistical computing. Viena: R Foundation for Statistical.

Reise, S. P., Moore, T. M., \& Haviland, M. G. (2010). Bifactor models and rotations: Exploring the extent to which multidimensional data yield univocal scale scores. Journal of Personality Assessment, 92(6), 544-559.

Rosseel, Y. (2012). lavaan: An R Package for Structural Equation Modeling. Journal of Statistical Software, 48(2), 1-36.

Sagarin, B. J. (2005). Reconsidering evolved sex differences in jealousy: Comment on Harris. Personality and Social Psychology Review, 9(1) 62-75. doi: 10.1207/s15327957pspr0901 5

Sagarin, B. J., \& Guadagno, R. E. (2004). Sex differences in the contexts of extreme jealousy. Personal Relationships, 11, 319-328. doi: 10.1111/j.1475-6811.2004.00085.x

Shackelford, T. K, Buss, D. M., \& Bennett, K. (2002). Forgiveness or breakup: Sex differences in responses to a partner's infidelity. Cognition and Emotion, 16(2), 299-307. doi: 10.1080/02699930143000202

Torres, A. R., Ramos-Cerqueira, A. T. de A., \& Dias, R. da S. (1999). O ciúme enquanto sintoma do transtorno obsessivo-compulsivo. Revista Brasileira de Psiquiatria, 21(3), 165-173. doi: 10.1590/S1516-44461999000300008

White, G. L. (1981). A model of romantic jealousy. Motivation and Emotion, 5(4), 295-310. doi: 10.1007/BF00992549

White, G. L., \& Mullen, P. E. (1992). Jealousy: Theory, research, and clinical strategies. New York: The Guilford Press.

World Health Organization. (2011). Standards and operational guidance for ethics review of health-related research. Geneva: WHO Document Production Services.

\section{Sobre os autores}

Hudson Fernandes Golino é Professor do Programa de Pós-Graduação em Computação Aplicada e da Graduação em Psicologia da Universidade Estadual de Feira de Santana.

Lucas de Francisco Carvalho é Professor do Programa de Pós-Graduação em Psicologia da Universidade São Francisco.

Elaine Silva é Graduanda do Curso de Psicologia da Faculdade de Tecnologia e Ciência.

Eliana Oliveira é Graduanda do Curso de Psicologia da Faculdade de Tecnologia e Ciência.

Sirneide Santos Musse é Graduanda do Curso de Psicologia da Faculdade de Tecnologia e Ciência.

Bruna Silva é Graduanda do Curso de Psicologia da Faculdade de Tecnologia e Ciência. 